\title{
BMJ Open Psychometric properties of the traditional Chinese version of the youth attitude to noise scale: a cross-sectional study in a large representative sample
}

\author{
Ningjing Chen (D) , Daniel Yee Tak Fong (D) , Sha Li (D) , Janet Yuen Ha Wong (D)
}

To cite: Chen N, Fong DYT, Li S, et al. Psychometric properties of the traditional Chinese version of the youth attitude to noise scale: a cross-sectional study in a large representative sample. BMJ Open 2021;11:e049722. doi:10.1136/ bmjopen-2021-049722

- Prepublication history for this paper is available online. To view these files, please visit the journal online (http://dx.doi. org/10.1136/bmjopen-2021049722).

Received 01 February 2021 Accepted 21 October 2021

Check for updates

(c) Author(s) (or their employer(s)) 2021. Re-use permitted under CC BY-NC. No commercial re-use. See rights and permissions. Published by BMJ.

School of Nursing, Li Ka Shing Faculty of Medicine, The University of Hong Kong, Hong Kong, China

Correspondence to Dr Daniel Yee Tak Fong; dytfong@hku.hk

\section{ABSTRACT}

Objectives This study aimed to examine the psychometric properties of the traditional Chinese version of the Youth Attitude to Noise Scale (YANS) in a large representative sample.

Design Cross-sectional study.

Setting 15 secondary schools in Hong Kong, China. Participants 2842 adolescents aged 12-20 years participated in this study between April and July 2016. Methods The standard forward-backward validation procedures were followed to obtain the traditional Chinese version of the YANS. Prior to the formal investigation, the YANS was evaluated by cognitive debriefing. The sample was randomly divided into two halves for exploratory factor analyses (EFAs) and confirmatory factor analyses (CFAs), respectively. The number of factors was determined by comparison data approach using EFAs, and the factor structure was confirmed by CFAs using the onefactor, four-factor and bifactor models. The scale's internal reliability, dimensionality and measurement invariance across gender and age groups were also examined. Results EFAs ( $n=1338)$ showed that four factors were extracted, and CFAs $(n=1337)$ demonstrated the bifactor model fitted better to the sample than the other models. Additionally, the traditional Chinese version of the YANS showed high reliability $(\omega=0.84)$, a general factor, scale multidimensionality, and gender and age invariance. Conclusions The findings of the current study indicate that the traditional Chinese version of the YANS is a feasible instrument to assess attitude to noise in Chinese adolescents, regardless of their gender and age. Given the presence of a general factor, the YANS is not merely multidimensional, and whether to use the total or subscale scores is recommended to rely on research objectives.

\section{INTRODUCTION}

As estimated by the WHO, nearly 1.1 billion young people are vulnerable to hearing loss due to loud noise exposure, including the unsafe use of personal listening devices. ${ }^{1}$ Due to noise exposure, tinnitus has troubled $5.4 \%-18.3 \%$ of adolescents ${ }^{2-4}$ and noise sensitivity has affected $17.1 \%-39.2 \%$ of adolescents. ${ }^{3} 4$ Sleep disturbance, ${ }^{5}$ noise annoyance ${ }^{6}$ and psychotropic drug
Strengths and limitations of this study

- This was the first study to evaluate the psychometric properties of the traditional Chinese version of the Youth Attitude to Noise Scale in a large-scale representative school sample.

- The number of factors was determined by a more accurate and robust comparison, namely the comparison data technique using exploratory factor analyses.

- The factor structure was confirmed by confirmatory factor analyses using the one-factor, four-factor and bifactor models.

- In this study, participants were only recruited from school settings, therefore the current results might not be applicable to the entire youth population.

- This study did not culturally adapt other scales to assess noise-related variables, which might affect adolescents' risk perception as well as attitude to noise.

utilisation ${ }^{7}$ have been identified as consequences of noise sensitivity.

Although the detrimental effects of noise exposure have been well illustrated, it is not sufficient for adolescents to enhance hearing protection in noisy environments. The main reason for this could be their attitude to noise. ${ }^{8}$ An individual with more positive attitude to noise considers noise as less annoying and feels less motivated to use hearing protection devices in noisy surroundings. ${ }^{9}$ Therefore, positive attitude to noise has been regarded as a key reason for loud sound exposure without hearing protection. ${ }^{9}$

Appropriate measurement of attitude to noise in adolescents is essential as it provides further empirical support for the implementation of effective interventions. One of the most effective instruments to measure attitude to noise is the Youth Attitude to Noise Scale (YANS), and its original version comprised 18 items specified with four factors, namely the 'Youth culture', 'Concentrate', 'Daily noises' 
and 'Intent to influence' factors. ${ }^{10}$ All items were rated on a 5-point Likert scale from 1 ('completely disagree') to 5 ('completely agree'), and a higher score reflects a more positive attitude to noise. ${ }^{10}$ Subsequently, a modified YANS with 19 items has been used in the USA, ${ }^{9}$ Brazil $^{11}$ and mainland China. ${ }^{12}$ However, although the aforementioned studies confirmed a four-factor structure, the specific contents in each factor were not identical across cultures $^{9-12}$ and several items displayed low factor loadings $(<0.4)$ necessitating removal. ${ }^{11}{ }^{12}$ Moreover, internal reliabilities estimated by Cronbach's alpha were fairly low $(<0.7)$ in some previous studies, particularly in the 'Concentrate'11 12 and 'Intent to influence' factors. ${ }^{12}$ In addition, although the other specific factors and the total YANS in a few studies showed an acceptable consistency, ${ }^{911} 12$ it is controversial to evaluate a scale's reliability by Cronbach's alpha due to the possible underestimation, and using other indices, for example, the omega $(\omega)$ coefficient provides a more precise estimate. ${ }^{13}$

Further, considering cultural diversities, using the simplified Chinese version of the YANS, it may be hard to unambiguously determine attitude to noise held by Chinese adolescents outside mainland China, for example, Hong Kong, Macao and Taiwan, where traditional Chinese is the official written language. Therefore, although the simplified Chinese version of the YANS has been recommended as a valid tool in youths from mainland China, ${ }^{12}$ there is no feasible version of the YANS that can be used outside mainland China.

In this context, another necessary concern regarding validation that has not been adequately examined yet is measurement invariance, which refers to the underlying constructs being examined equivalently across groups. ${ }^{14}$ This procedure is also particularly crucial in adolescents given that during adolescence, enormous physical and psychological changes occur concurrently and manifest distinctively by gender and age. For this reason, examining the gender and age invariance of the YANS enables us to confirm whether the latent constructs of the YANS are measured similarly across gender and age groups.

Given the knowledge gaps and limitations of the existing research, the current study aimed to examine the psychometric properties of the traditional Chinese version of the YANS in a representative school sample. Specifically, the objectives were: (1) to explore the factor structure of the traditional Chinese version of the YANS by exploratory factor analyses (EFAs), (2) to confirm the underlying scale structure by confirmatory factor analyses (CFAs) using the one-factor, four-factor and bifactor models, (3) to assess the scale's internal reliability, dimensionality, and measurement invariance across gender and age groups.

\section{METHODS}

\section{Study design}

This was a cross-sectional study.

\section{Participants}

Adolescence is divided into young (10-14 years), middle (15-19 years) and late (20-24 years) stages. ${ }^{16}$ Therefore, students aged 10-24 years and in grades 7-12 in Hong Kong were included and those who could not read traditional Chinese or had doctor-diagnosed hearing impairment were excluded. The sample size calculation was originally based on the estimation of the prevalence of noise exposure. With an anticipated prevalence of $5 \%$ and a $1 \%$ margin of error in a $95 \%$ CI, 1825 participants were needed. ${ }^{17}$ After accounting for incomplete responses, 2000 participants were targeted. In this study, the sample was randomly divided into two halves to conduct EFAs and CFAs, respectively. Adopting the rule of 20 participants per item for factor analysis, ${ }^{18}$ validating the modified 19-item YANS required 760 participants. Thus, 2000 participants were adequate.

\section{Cultural adaptation to the YANS}

We followed the standard forward-backward validation procedures ${ }^{19}$ where two bilingual registered nurses independently translated the original YANS into traditional Chinese. In a consensus meeting consisting of these two translators and a researcher with prior experience in cross-cultural scale adaptations, ${ }^{20}$ differences in these two Chinese versions were discussed and a consensus Chinese version was obtained. Another bilingual registered nurse who was not aware of the original English version translated the consensus Chinese version into the English version. The backward and original English versions were then compared. Therefore, a modified Chinese version was obtained and evaluated by cognitive debriefing in five Chinese adolescents with a mean age of 15.80 years ( $\mathrm{SD}=2.28$; range $13-18$ years $)$. The average completion time was $2.30 \mathrm{~min}(\mathrm{SD}=0.67$; range $1.5-3.0 \mathrm{~min})$. The median rating of the scale length was acceptable, and that for item clarity as well as relevance was high on a 5-point Likert scale, where $0=$ 'very short/very low' and $4=$ "very long/very high'.

Participants were invited to rate how they agreed with the statements regarding their attitude to noise. Items 1 , $3,4,7,8,12,13,15,18$ and 19 were scored inversely in the modified 19-item YANS. ${ }^{210}$ However, as the item 8 'Do not like quiet' reveals a positive attitude to noise, items 1 , $3,4,7,12,13,15,18$ and 19 were scored inversely in this study. The sum of the total YANS score ranged from 19 to 85 , with a higher YANS score indicating more positive attitude to noise.

Additionally, participants' sociodemographic information, including their age, gender and study grade, was also collected.

\section{Procedures}

This study was conducted between April and July 2016. Based on the list of public secondary schools available from the Hong Kong Education Bureau, one secondary school in each district was selected randomly and sent an invitation letter. Finally, 15 schools were recruited in 
our study. Before the day when students participated, we explained the study procedures in detail to their parents and required them to give back a signed note if they disagreed with their children's participation. Students were also informed of the study details and signed an informed consent form before they engaged. Teachers in the schools helped us to disseminate the questionnaires, and it took each student around $25 \mathrm{~min}$ to complete.

\section{Statistical analyses}

\section{Summary}

In the entire dataset, missing values were replaced by the mean values of the observed item responses in each case, where no less than $50 \%$ of items were answered. ${ }^{20}$ The total score and each subscale score were standardised onto a $0-100$ scale, respectively. ${ }^{20}$ In addition, the floor and ceiling effects were also calculated. A percentage of less than $15 \%$ was considered as no substantial floor or ceiling effects. ${ }^{21}$

Descriptive statistics were used to summarise the sample characteristics. To examine the factor structure of the YANS, the sample was randomly divided into two halves, with one half serving as the training set for EFAs, and the other half serving as the validation set for CFAs. ${ }^{20}$ General analyses and EFAs were conducted in IBM SPSS V.25.0, while CFAs were performed in RStudio V.1.2.5042 using package 'lavaan'.22 The nominal level of significance was set as 0.05 in all statistical analyses.

\section{Exploratory factor analyses}

EFAs were conducted using maximum likelihood estimation with promax rotation. The number of factors was determined using the comparison data technique, which has been proven to be more accurate and robust than the other methods. ${ }^{20}{ }^{23}$ Any item with communality less than 0.10 or a factor loading of magnitude smaller than 0.40 was removed. ${ }^{20} 24$

\section{Confirmatory factor analyses}

Subsequently, CFAs were conducted using the validation set to evaluate the fitness of the factor structure derived from EFAs. Item responses were regarded as ordinal data, and the diagonally weighted least squares estimator was selected. In a bifactor model, the general factor is restricted to be uncorrelated with specific factors, whereas specific factors can intercorrelate or not. ${ }^{25}{ }^{26}$ Given that the correlations among the specific factors of the YANS possibly existed, the specific factors were allowed to intercorrelate in the bifactor model in this study. In accordance with the theoretical framework related to attitude to noise, three models were assessed: (1) the one-factor model, (2) the four-factor model and (3) the bifactor model (figure 1).

As $\chi^{2}$ statistic shows high sensitivity to a large sample, ${ }^{27}$ the goodness-of-fit of the CFA models was measured by the root mean square error of approximation (RMSEA), standardised root mean square residual (SRMR),
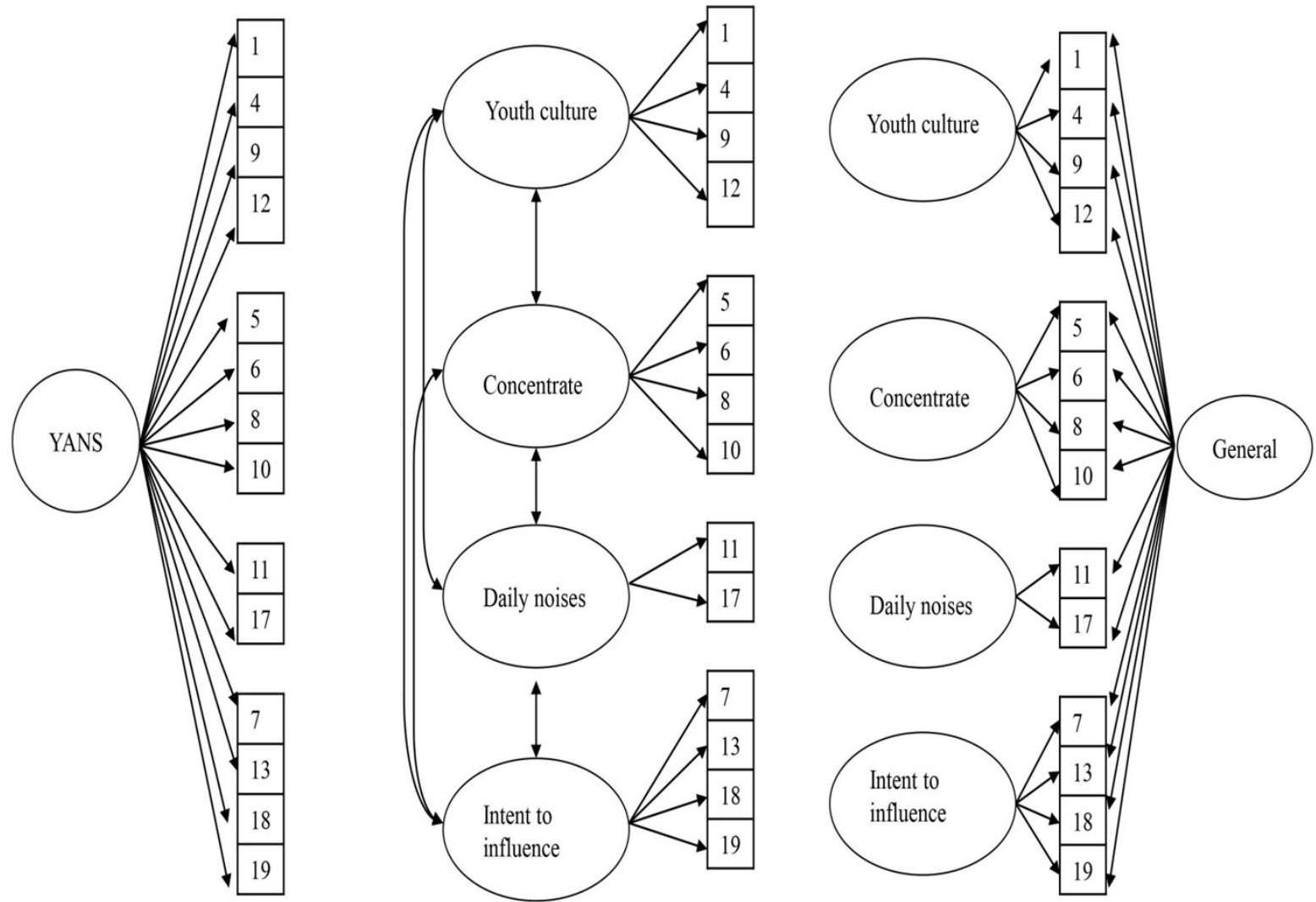

Figure 1 The one-factor, four-factor and bifactor models of the traditional Chinese version of the YANS. YANS, Youth Attitude to Noise Scale. 
comparative fit index (CFI) and the Tucker-Lewis Index (TLI) ${ }^{28}$ A CFA model is adequately fitted when RMSEA and SRMR $<0.08$, whereas CFI and TLI $>0.95$. ${ }^{28} 29$

\section{Examination of internal reliability}

The internal reliability was examined by the $\omega$ coefficient, ${ }^{3031}$ which conquers deficiencies of Cronbach's alpha given the assumption of tau equivalence. ${ }^{15}$ An $\omega$ value of at least 0.70 for the general factor indicates a reliable total score. ${ }^{32}$ Likewise, the $\omega$ value for a subscale $\left(\omega_{\mathrm{s}}\right)$ should be above 0.50 , and a preferred value is approximately 0.75 , indicating a reliable subscale score after controlling the effects of the general factor. ${ }^{33}$ Moreover, the $\omega$ hierarchical $\left(\omega_{\mathrm{H}}\right)$ value of the total scale was calculated to determine the degree of total score variance that could be explained by the general factor. ${ }^{33}$ The $\omega_{\mathrm{H}}$ value for the general factor greater than 0.50 suggests a meaningful description, and that greater than 0.80 indicates essential scale unidimensionality. ${ }^{24}{ }^{34}$ In addition, the $\omega_{\mathrm{H}}$ for a subscale $\left(\omega_{\mathrm{HS}}\right)$ reflects the proportion of subscale score variance that can be attributed to the specific subscale, and a moderately substantial value of $\omega_{\mathrm{HS}}$ is suggested to be at least $0.20 .{ }^{35}$ Moreover, a higher value of $\omega_{\mathrm{S}}$ than $\omega_{\mathrm{HS}}$ in each subscale implies that a considerable proportion of subscale scores variance could be explained by the general factor rather than the specific factors. ${ }^{36}$

\section{Evaluation of scale dimensionality}

The standardised factor loadings for the one-factor, fourfactor and bifactor models were also estimated. Unidimensionality can be concluded when the factor loadings of the general factor in the bifactor model are higher than those in the one-factor model, whereas the factor loadings of the specific factors in the bifactor model are lower than those in the four-factor model. ${ }^{37}$ In contrast, lower factor loadings of the general factor in the bifactor model than those in the one-factor model and higher factor loadings of the specific factors in the bifactor model than those in the four-factor model provide evidence for multidimensionality ${ }^{37}$ Further, the explained common variance (ECV) is regarded as the quotient of the division of the explained variance attributed to the general factor by the variance attributed to the general and specific factors. ${ }^{38}$ An ECV value of 0.50 implies that the general and specific factors equally account for the common variance, ${ }^{38}$ and an ECV value of at least 0.70 supports a unidimensional model. ${ }^{24}$ In addition to the $\mathrm{ECV}$, the percentage of uncontaminated correlations (PUC), which refers to "the number of uncontaminated correlations divided by the number of unique correlations, ${ }^{39}$ is another indicator of dimensionality. Both ECV and PUC are suggested to be greater than 0.70 , which indicates a unidimensional model. ${ }^{36}$ Nevertheless, when the PUC value is smaller than 0.80 , the cut-off points 0.60 for the ECV and 0.70 for the $\omega_{\mathrm{H}}$ for the general factor should be selected to evaluate a unidimensional model. ${ }^{2440}$

Additionally, the $\mathrm{H}$ index was used to measure the construct replicability, and an $\mathrm{H}$ index value above 0.80 reveals a well-defined latent variable. ${ }^{36}$ In this study, bifactor statistical indices were calculated using the package 'BifactorIndicesCalculator'. ${ }^{41}$

\section{Measurement invariance analyses}

We further assessed measurement invariance for the bestfitting model by gender and age using multiple group CFA models. Comparing two nested models, a change of RMSEA $(\triangle$ RMSEA $)<0.015$, a change of SRMR $(\triangle$ SRMR $)$ $<0.030$ and a change of CFI $(\triangle \mathrm{CFI})<0.010$ suggested invariance. $^{42}$

\section{Patient and public involvement}

There was no patient or public involvement in this study.

\section{RESULTS}

\section{Sample characteristics}

A total of 2842 participants were recruited in this study. In our sample, 167 participants did not answer all items of the YANS but responded to more than $50 \%$ items of the YANS. Therefore, they were not included in the factor analyses. The remaining sample of 2675 participants was spilled randomly, with 1338 participants in the training set for EFAs and 1337 participants in the validation set for CFAs, respectively. In the entire sample, participants were aged between 12 and 20 years, with a mean age of 15.24 years $(\mathrm{SD}=1.59)$, and $55.2 \%$ of participants were female. Participants were almost equally distributed in each grade. As indicated in table 1, gender and age differences between the factor and non-factor analysis sample were insignificant $(\mathrm{p}>0.05)$.

\section{Exploring the factor structure of the YANS}

The training set comprised 1338 participants, and comparison data method indicated that four factors explained $35.9 \%$ of total variance would be optimal. Using maximum likelihood estimation to extract factors, items Q2, Q3, Q14, Q15 and Q16 with rotated factor loadings less than 0.4 or communalities less than 0.1 were removed.

\section{Confirming the factor structure of the YANS}

Table 2 shows CFA fit indices for the one-factor, four-factor and bifactor models of the YANS. Overall, the one-factor model did not exhibit satisfactory fits. The four-factor model adequately fitted to the sample, better than the one-factor model. Nevertheless, the bifactor model fitted best to the sample among the three models.

\section{Factor intercorrelations}

Generally, regarding the correlated four-factor model, significant and moderate-to-strong correlations were observed among the latent constructs. All correlation coefficients were no smaller than $0.30(\mathrm{p}<0.05)$ with two exceptions, the correlation between the 'Daily noises' and 'Youth culture' factors being $0.18(\mathrm{p}<0.05)$ and between the 'Daily noises' and 'Intent to influence' factors being $0.11(\mathrm{p}<0.05)$. 
Table 1 Characteristics of the Chinese adolescents

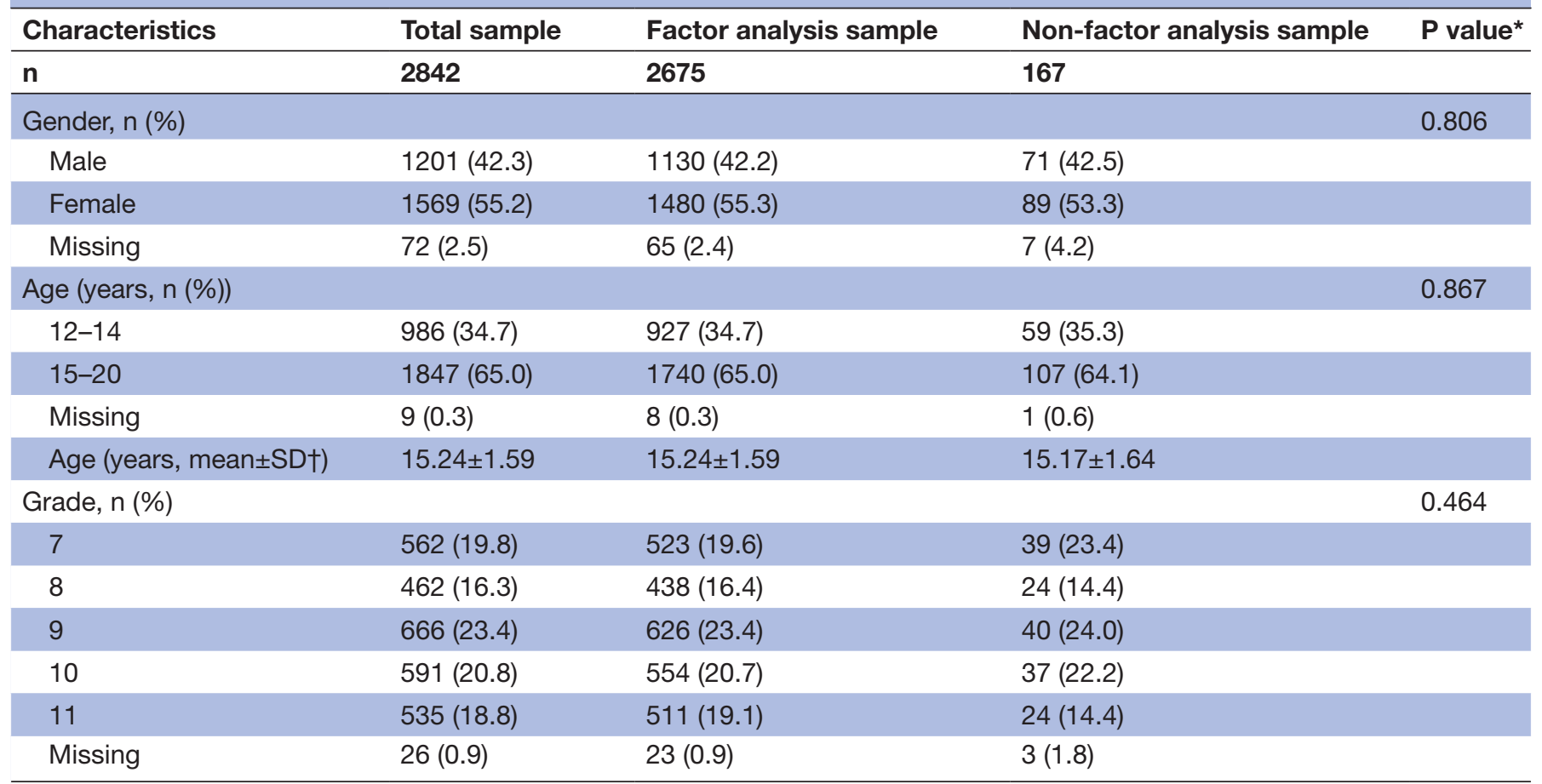

*Estimated by Pearson's $\mathrm{X}^{2}$ test and non-missing data in both factor and non-factor analysis samples. †The mean age (SD) was calculated by excluding missing values.

\section{Internal reliability}

The $\omega$ for the general factor was 0.84 , indicating high reliability (table 3 ). The $\omega_{\mathrm{s}}$ was between 0.62 and 0.85 , revealing moderate-to-high reliability. The $\omega_{H}$ for the general factor was 0.65 , which was slightly lower than the cut-off value of $0.80 .{ }^{2434}$ The $\omega_{\mathrm{HS}}$ was moderate to substantial in all subscales except for the 'Concentrate' factor with a low $\omega_{\mathrm{HS}}$ value of 0.06 .

\section{Standardised factor loadings and dimensionality}

Table 3 displays the standardised factor loadings for the one-factor, four-factor and bifactor models. Overall, the standardised factor loadings of the general factor in the bifactor model were comparable with those in the onefactor model. Mean absolute difference of factor loadings was 0.09 ( $\mathrm{SD}=0.07$; ranging between 0.01 and 0.24 ). Additionally, the standardised factor loadings of the specific factors in the bifactor model were considerably lower than those in the four-factor model. Mean absolute difference of factor loadings was 0.30 ( $\mathrm{SD}=0.21$; ranging between 0.05 and 0.72 ). This was the first evidence that revealed the presence of a general factor. Additionally, a high $\omega$ value was found in the general factor (ie, 0.84 ), demonstrating a reliable total score. Moreover, all $\omega_{\mathrm{s}}$ values were higher than $\omega_{\mathrm{HS}}$ implying that the major subscale score variance was accounted for by the general factor. In addition, only the general factor had an $\mathrm{H}$ index value above 0.80 , indicating the strength of a stable general factor. All these results, together with moderateto-strong intercorrelations of latent factors in the fourfactor model, pointed out that the general factor in the bifactor model had a significant impact on the common variance.

Table 2 Fit indices for the CFA models of the YANS in Chinese adolescents $(n=1337)$

\begin{tabular}{llll}
\hline Fit indices & One-factor model & Four-factor model & Bifactor model* $^{*}$ \\
\hline$\chi^{2}(\mathrm{df})$ & $2780.3(77)$ & $644.3(71)$ & $215.8(57)$ \\
RMSEA $(90 \% \mathrm{Cl})$ & $0.162(0.157$ to 0.167$)$ & $0.078(0.072$ to 0.083$)$ & $0.046(0.039$ to 0.052$)$ \\
SRMR & 0.109 & 0.058 & 0.034 \\
CFI & 0.842 & 0.967 & 0.991 \\
TLI & 0.814 & 0.957 & 0.985 \\
\hline
\end{tabular}

*Given that the correlations among the group factors possibly existed, the group factors were allowed to intercorrelate in the bifactor model. CFA, confirmatory factor analysis; CFI, comparative fit index; RMSEA, root mean square error of approximation; SRMR, standardised root mean square residual; TLI, Tucker-Lewis Index; YANS, Youth Attitude to Noise Scale. 


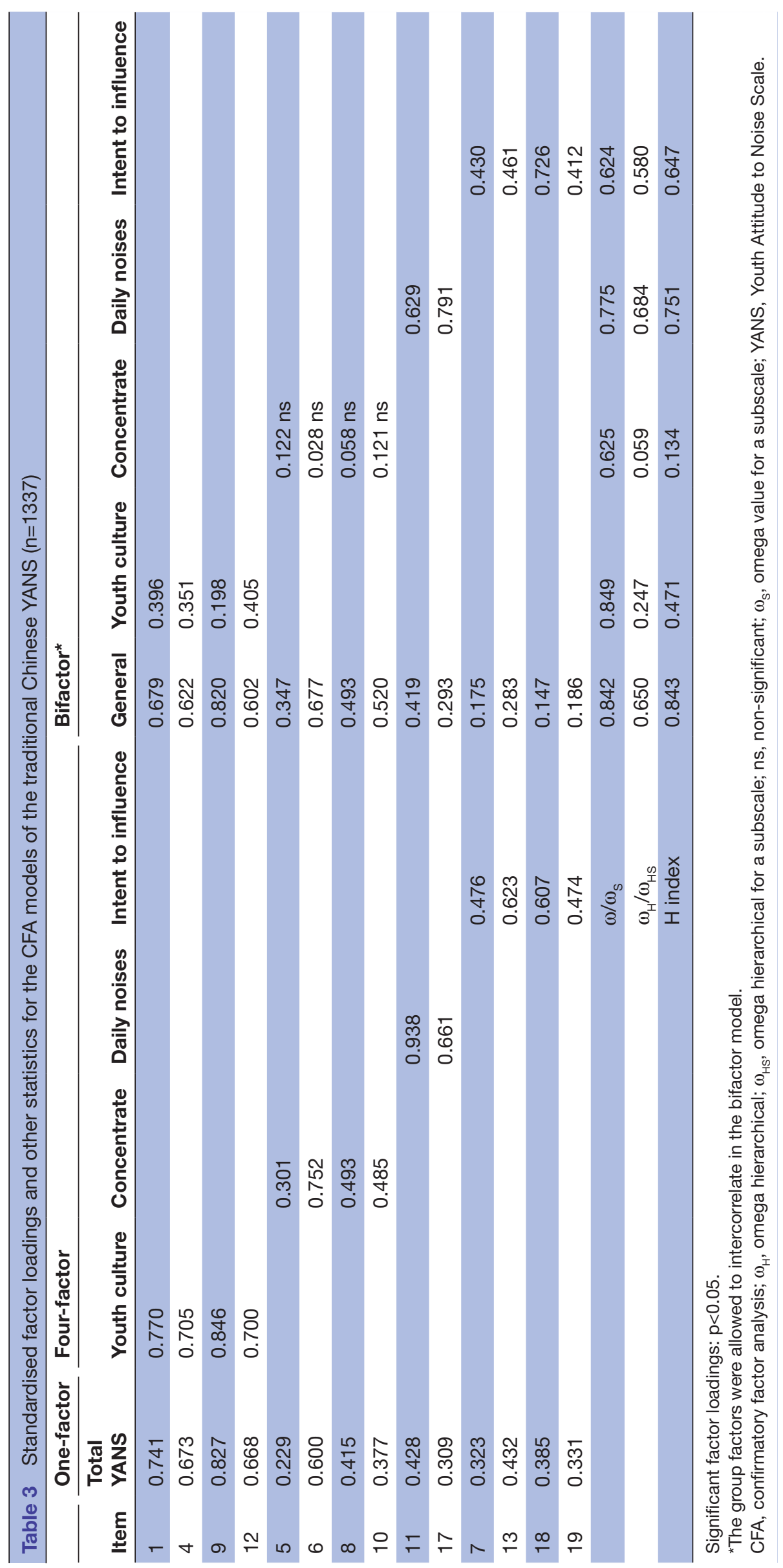


On the other hand, all the items loaded strongly $(>0.40)$ on the specific factors of the four-factor model with only an exception of item $5(0.30)$, which provided an indication of a multidimensional model. Moreover, the values of PUC, ECV and $\omega_{\mathrm{H}}$ for the general factor were 0.79 $(<0.80), 0.49(<0.60)$ and $0.65(<0.70)$, respectively. Given that all $\omega_{\mathrm{S}}$ values were greater than the acceptable criteria of $0.50,{ }^{33}$ in combination with moderately substantial $\omega_{\mathrm{HS}}$ values for the three subscales, which failed to support essential unidimensionality of the YANS, a multidimensional model should be considered. In the same vein, high $\mathrm{H}$ index values were also found for the 'Daily noises' and 'Intent to influence' factors (ie, 0.75 and 0.65 , respectively), and thus the scale multidimensionality was recommended.

\section{Scale summary}

Participants had a mean total score of 44.37 (SD=13.00). The total YANS reported no ceiling and a $0.1 \%$ floor rate. The floor and ceiling percentages in the subscales were $1.5 \%-4.2 \%$ and $0.7 \%-2.8 \%$, respectively. This result revealed that although the total scale and subscales differed in item responses, they did not demonstrate floor and ceiling effects.

\section{Measurement invariance}

As there were just 13 participants $(0.5 \%)$ aged 20 years, we assessed measurement invariance only between 12-14 years and 15-20 years age group. Generally, the bifactor models satisfactorily fitted to the data in age and gender subgroups and demonstrated measurement invariance. Specifically, indices values were RMSEA $\leq 0.055$, SRMR $\leq 0.042$, CFI $\geq 0.982$ and $T L I \geq 0.975$ (table 4) in all subgroups. Regarding participants' gender, configural invariance was well established (RMSEA $=0.051$, $\mathrm{SRMR}=0.038$, CFI $=0.988$, TLI $=0.980)$ and metric invariance was found $(\triangle$ RMSEA $\leq 0.002, \triangle$ SRMR $\leq 0.004$ and $\triangle$ CFI $\leq 0.004)$. Further, scalar invariance was determined $(\triangle$ RMSEA $\leq 0.003, \triangle$ SRMR $\leq 0.001$ and $\triangle$ CFI $\leq 0.002)$. This provided valid evidence that the latent constructs of the YANS were examined similarly between male and female adolescents. Correspondingly, configural, metric and scalar invariances in both 11-14 years and 15-20 years age groups were confirmed as well, as the fit index changes between nested models did not exceed the recommended benchmarks $(\triangle$ RMSEA $<0.015, \triangle$ SRMR $<0.030$ and $\triangle \mathrm{CFI}<0.010) .{ }^{42}$

\section{DISCUSSION}

To our knowledge, this is the first study to evaluate the psychometric properties of the traditional Chinese version of the YANS in a large-scale representative school sample. Specifically, EFA results suggested that 14 items loaded on four factors should be retained. Subsequently, we confirmed the factor structure of the YANS by performing one-factor, four-factor and bifactor models. Factor loadings of these three models in conjunction with the bifactor statistical indices showed a general factor of the YANS, scale multidimensionality and high-scale internal reliabilities. Lastly, our research findings showed that the YANS had no floor and ceiling effects and was measurement invariant across age and gender groups.

The evidence for a general factor of the YANS could be found from similar factor loadings on the general factor

Table 4 Gender and age invariance for the bifactor model of the traditional Chinese YANS

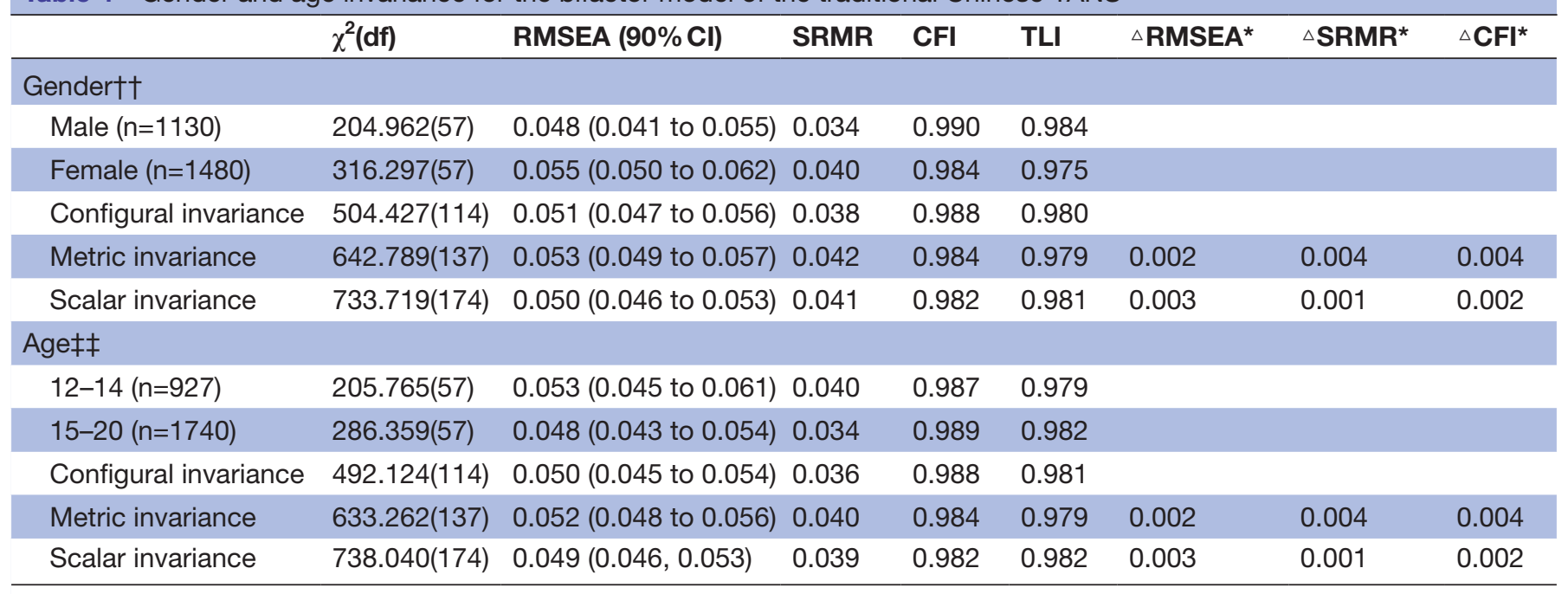

\footnotetext{
${ }^{*}$ The absolute value was reported.

†The gender invariance analysis using the study sample, excluding 65 cases with missing value in the gender variable.

$\ddagger$ The age invariance analysis also using the study sample, excluding eight cases with missing value in the age variable.

$\triangle \mathrm{CFI}$, changes of $\mathrm{CFI}$ in comparison with the prior less restrained model; CFI, comparative fit index; $\triangle \mathrm{RMSEA}$, changes of RMSEA in comparison with the prior less restrained model; RMSEA, root mean square error of approximation; $\triangle$ SRMR, changes of SRMR in comparison with the prior less restrained model; SRMR, standardised root mean square residual; TLI, Tucker-Lewis Index; YANS, Youth Attitude to Noise Scale.
} 
in the bifactor model to those of the one-factor model. An additional indication for a general factor was that items loaded less strongly on the specific factors in the bifactor model than in the four-factor model. In addition, a high $\omega$ value was found in the general factor (ie, 0.84), which was indicative of a reliable total score. Moreover, a higher value of $\omega_{\mathrm{S}}$ than $\omega_{\mathrm{HS}}$ was found in each subscale, which further enhanced the presence of a general factor. Additional evidence for a general factor was the high $\mathrm{H}$ index value $(>0.80)$ only found for the general factor, pointing to the general factor with a well-defined definition that could be measured precisely by its response items, ${ }^{36}$ which again supported the strength of a stable general factor. Furthermore, regarding the correlated four-factor model, latent factors were intercorrelated moderately to strongly, except for the less strong correlation coefficients between the 'Daily noises' and 'Youth culture' factors and between the 'Daily noises' and 'Intent to influence' factors, which were similar to the findings reported by Widen $e t a t^{\dagger}$ and Zhu $e t a l{ }^{12}$ Although the less strong coefficients might be explained by the small number (ie, two) of items with salient factor loadings on the 'Daily noises' factor, this result indicated that although each factor best represented a specific meta-construct of attitude to noise, a common underlying construct was shared among factors. Combining these findings, it was reasonable to conclude that the general factor had substantial effects on the overall variance of the YANS.

On the other hand, there was sufficient evidence supporting that the constructs of the YANS were conceptualised as multidimensional. First, prior to EFAs, four factors were extracted. Second, after removing 5 items loaded poorly onto the specific factors, 14 retained items had strong factor loadings $(>0.40)$ and adequate communalities $(>0.10)$. This finding was confirmed by the standardised factor loadings for the correlated four-factor model. Although item 5 had a less strong factor loading on the 'Concentrate' factor (ie, 0.30), all the other retained items loaded strongly on the specific factors with standardised factor loadings ranging from 0.47 to 0.94 . Third, although the bifactor model showed a good fit to the data, the ECV in the present study was somewhat low. Given that PUC was below 0.80 , the benchmarks for ECV above 0.60 and $\omega_{\mathrm{H}}$ for the general factor above 0.70 were used to confirm a unidimensional model. ${ }^{40}$ In the present study, given the values of PUC being 0.79, ECV being 0.49 as well as $\omega_{\mathrm{H}}$ for the general factor being 0.65 , a four-factor model should be proposed. Fourth, the relatively high $\omega$ values were also identified in the 'Youth culture' $(0.85)$, 'Daily noises' (0.78), 'Concentrate' (0.63) and 'Intent to influence' factors $(0.62)$, which were all higher than the benchmark $0.50,{ }^{33}$ representing reliable subscale scores of distinct dimensions. Therefore, taken together with approximately $50 \%$ of variance that could be explained by the specific factors rather than the general factor, the uncertainty to calculate the YANS score from separate subscales was reduced. Further evidence for a multidimensional model was detected from moderately substantial values of $\omega_{\mathrm{HS}}(\geq 0.20) .{ }^{41}$ In the current study, the $\omega_{\mathrm{HS}}$ values were moderately substantial in all subscales except for the 'Concentrate' factor with a low $\omega_{\mathrm{H}}$ value of 0.06 . Thus, it was reasonable to conclude a multidimensional model. Lastly, similar to the general factor, the $\mathrm{H}$ index values of the two specific factors, namely the 'Daily noises' and 'Intent to influence' factors, were also relatively high. This illustrated that to some extent, latent variables could be described precisely by the subscale scores. ${ }^{36}$

Given that a general factor of the YANS was identified, although the multidimensional nature of the scale was determined, it was not recommended to evaluate subscale scores in each case due to the potential confounding relationships of the general factor with the specific factors using modelling approaches other than the bifactor analysis, in which confounding effects were removed. ${ }^{15}$ Therefore, we suggested that the determination should depend on research interest. Under some conditions, although it might be encouraging to use the total YANS score rather than the subscale scores, an important aspect was that the overall variance of the total score consisted of not only the variance accounted for by the general factor but also the variance explained by the specific factors. As such, it was essential to present the means of the total YANS score and its associations with other latent variables with caution..$^{30}$ Additionally, for further use of the scale, another necessary task was to externally validate the YANS using a bifactor structure within structural equation modelling and to investigate the significant conceptual associations among the general and specific factors of the YANS as well as other predicted variables, for instance, hearing protection. ${ }^{9}$

Although the current study advances our understanding of the psychometric properties of the YANS, there are several limitations. First, participants were only recruited from school settings, which warranted sample homogeneity. However, the current results might not be applicable to the entire youth population, and future research may recruit more diverse samples from various settings (eg, clinical or community settings). Second, regarding the age range, adolescence lasts between 10 and 24 years. However, our participants were 12-20 years, which again limited our study findings to represent those of all the adolescents. Third, this study did not culturally adapt other scales to assess noise-related variables, for example, noise sensitivity ${ }^{3}$ and noise annoyance, ${ }^{44}$ which might affect adolescents' risk perception as well as attitude to noise. Therefore, it will be worthy for future studies to explore the associations among attitude to noise, noise sensitivity and noise annoyance by using separate scales, for instance, the YANS, the ${ }^{20}$ Weinstein's Noise Sensitivity Scale and the Annoyance Index ${ }^{44}$ to strengthen the applicability of the YANS. Fourth, we did not investigate the test-retest reliability of the YANS, which is suggested to be tested in future studies to have a comprehensive understanding of the psychometric nature of the YANS. Lastly, regarding a bifactor model, it is more likely to exhibit satisfactory fits than the other models. ${ }^{37}$ As such, it should 
not be neglected that the bifactor analysis may build an overfit model as a result of its innate traits. Nevertheless, the bifactor approach enables us to identify whether there is a general factor that can be precisely described by the total score or whether it is plausible to use the subscale scores. $^{37}$

\section{CONCLUSIONS}

The traditional Chinese version of the YANS is a feasible instrument to assess attitude to noise in Chinese adolescents, regardless of their gender and age. Although the YANS is primarily multidimensional in nature, the general factor accounted for a considerable proportion of total variance. Therefore, the constructs of the YANS are not merely multidimensional, and whether to use the total or subscale scores should rely on research interest. Future research is also suggested to assess the relationships among the general and specific factors of the YANS as well as other predicted variables by structural equation modelling, which will expand the literature by adding more important information provided by the general and specific factors.

\section{Acknowledgements The authors thank all the participants and their parents.}

Contributors DYTF conceptualised the study. DYTF, SL and JYHW collected data. $\mathrm{NC}$ analysed the data and DYTF provided suggestions on the data analysis. NC wrote the first manuscript and all authors commented on the first manuscript. NC revised the manuscript, and all authors read and approved the final manuscript. DYTF is the guarantor of this study.

Funding The study was supported by the Small Project from The University of Hong Kong (grant number 201409176046).

Competing interests None declared.

Patient and public involvement Patients and/or the public were not involved in the design, or conduct, or reporting, or dissemination plans of this research.

\section{Patient consent for publication Not required.}

Ethics approval The study protocol was granted by the Institutional Review Board of the University of Hong Kong/Hospital Authority Hong Kong West Cluster (reference number: UW 15-380), and informed consent was obtained from all the participants and their parents.

Provenance and peer review Not commissioned; externally peer reviewed.

Data availability statement Data are available upon reasonable request. The data used to support the findings of this study are available from the corresponding author upon reasonable request.

Open access This is an open access article distributed in accordance with the Creative Commons Attribution Non Commercial (CC BY-NC 4.0) license, which permits others to distribute, remix, adapt, build upon this work non-commercially, and license their derivative works on different terms, provided the original work is properly cited, appropriate credit is given, any changes made indicated, and the use is non-commercial. See: http://creativecommons.org/licenses/by-nc/4.0/.

\section{ORCID iDs}

Ningjing Chen http://orcid.org/0000-0002-5419-8505

Daniel Yee Tak Fong http://orcid.org/0000-0001-7365-9146

Sha Li http://orcid.org/0000-0001-6238-7544

Janet Yuen Ha Wong http://orcid.org/0000-0002-3000-4577

\section{REFERENCES}

1 World Health Organization. New WHO-ITU standard aims to prevent hearing loss among 1.1 billion young people, 2021. Available: https:// www.who.int/news/item/12-02-2019-new-who-itu-standard-aims-toprevent-hearing-loss-among-1.1-billion-young-people
2 Gilles A, Van Hal G, De Ridder D, et al. Epidemiology of noiseinduced tinnitus and the attitudes and beliefs towards noise and hearing protection in adolescents. PLoS One 2013;8:e70297.

3 Widén SEO, Erlandsson SI. Self-Reported tinnitus and noise sensitivity among adolescents in Sweden. Noise Health 2004;7:29-40.

4 Landälv D, Malmström L, Widén SE. Adolescents' reported hearing symptoms and attitudes toward loud music. Noise Health 2013;15:347-54.

5 Howell DR, Oldham JR, Brilliant AN, et al. Trouble falling asleep after concussion is associated with higher symptom burden among children and adolescents. J Child Neurol 2019;34:256-61.

6 Sung JH, Lee J, Jeong KS, et al. Influence of transportation noise and noise sensitivity on annoyance: a cross-sectional study in South Korea. Int J Environ Res Public Health 2017;14:322.

7 Okokon EO, Yli-Tuomi T, Turunen AW, et al. Traffic noise, noise annoyance and psychotropic medication use. Environ Int 2018; $119: 287-94$

8 Lee D, Han W. Noise levels at baseball stadiums and the spectators' attitude to noise. Noise Health 2019;21:47-54.

9 Widén SE, Holmes AE, Erlandsson SI. Reported hearing protection use in young adults from Sweden and the USA: effects of attitude and gender. Int J Audiol 2006;45:273-80.

10 Olsen SE. Psychological aspects of adolescents' perceptions and habits in noisy environments. Göteborg University 2004.

11 Zocoli AMF, Morata TC, Marques JM. Youth attitude to noise scale (YANS) questionnaire adaptation into Brazilian Portuguese. Braz $J$ Otorhinolaryngol 2009;75:485-92.

12 Zhu X, Bihi A, Hu X, et al. Chinese-adapted youth attitude to noise scale: evaluation of validity and reliability. Noise Health 2014;16:218-22.

13 Español-Martín G, Pagerols M, Prat R, et al. Strengths and difficulties questionnaire: psychometric properties and normative data for Spanish 5- to 17-year-olds. Assessment 2021;28:1445-58.

14 Finch $\mathrm{H}$. Encyclopedia of quality of life and well-being research: measurement invariance, 2020. Available: https://link.springer.com/ referenceworkentry/10.1007\%2F978-94-007-0753-5_1759

15 Zwaanswijk W, Veen VC, Vedder P. The youth psychopathic traits inventory: a bifactor model, dimensionality, and measurement invariance. Assessment 2017;24:932-44.

16 Kinghorn A, Shanaube K, Toska E, et al. Defining adolescence: priorities from a global health perspective. Lancet Child Adolesc Health 2018;2:e10.

17 Chen N, Fong DYT, Li S, et al. Association between non-restorative sleep and quality of life in Chinese adolescents. Int J Environ Res Public Health 2020;17:7249.

18 Teese R, Willie C, Jago A, et al. An investigation of alternative factor models of impulsivity using the UPPS-P. J Pers Assess 2021;103:324-31.

19 Beaton DE, Bombardier C, Guillemin F, et al. Guidelines for the process of cross-cultural adaptation of self-report measures. Spine 2000;25:3186-91.

20 Fong DYT, Takemura N, Chau P-H, et al. Measurement properties of the Chinese Weinstein noise sensitivity scale. Noise Health 2017; 19:193-9.

$21 \mathrm{Gu}$ J, Baer R, Cavanagh K, et al. Development and psychometric properties of the Sussex-Oxford compassion scales (SOCS). Assessment 2020;27:3-20.

22 Rosseel Y, Jorgensen TD, Rockwood N, et al. Package 'lavaan'. R package version 0.6-6, 2020. Available: https://cran.r-project.org/ web/packages/lavaan/lavaan.pdf

23 Ruscio J, Roche B. Determining the number of factors to retain in an exploratory factor analysis using comparison data of known factorial structure. Psychol Assess 2012;24:282-92.

24 Pirani S, Kulhanek C, Wainwright K, et al. The reasons for living inventory for young adults (RFL-YA-II). Assessment 2021;28:942-54.

25 Reise SP, Bonifay WE, Haviland MG. Bifactor modelling and the evaluation of scale scores. US, Hoboken: John Wiley \& Sons inc, 2018: 677-708.

26 Dunn KJ, McCray G. The place of the bifactor model in confirmatory factor analysis investigations into construct dimensionality in language testing. Front Psychol 2020;11:1357.

27 van de Schoot R, Lugtig P, Hox J. A checklist for testing measurement invariance. Eur J Dev Psychol 2012;9:486-92.

28 LT H, Bentler PM. Fit indices in covariance structure modeling: sensitivity to underparameterized model misspecification. Psychol Methods 1998;3:424-53.

29 LT H, Bentler PM. Cutoff criteria for fit indexes in covariance structure analysis: conventional criteria versus new alternatives. Structural Equation Modeling: A Multidisciplinary Journal 1999;6:1-55. 
30 Reis D, Lehr D, Heber E, et al. The German version of the perceived stress scale (PSS-10): evaluation of dimensionality, validity, and measurement invariance with exploratory and confirmatory bifactor modeling. Assessment 2019;26:1246-59.

31 Zanon C, Brenner RE, Baptista MN. Examining the dimensionality, reliability, and invariance of the depression, anxiety, and stress Scale-21 (DASS-21) across eight countries. Assessment 2020:107319111988744-49.

32 Grygiel P, Humenny G, Rębisz S. Using the de Jong Gierveld loneliness scale with early adolescents: factor structure, reliability, stability, and external validity. Assessment 2019;26:151-65.

33 Reise SP, Bonifay WE, Haviland MG. Scoring and modeling psychological measures in the presence of multidimensionality. $J$ Pers Assess 2013;95:129-40.

34 Arias VB, Ponce FP, Núñez DE. Bifactor models of attention-deficit/ hyperactivity disorder (ADHD): an evaluation of three necessary but underused psychometric indexes. Assessment 2018;25:885-97.

35 Smits IAM, Timmerman ME, Barelds DPH. The Dutch symptom Checklist-90-Revised: is the use of the subscales justified? European Journal of Psychological Assessment 2015;31:263-71.

36 Rodriguez A, Reise SP, Haviland MG. Applying bifactor statistical indices in the evaluation of psychological measures. J Pers Assess 2016:98:223-37.

37 Monteiro F, Fonseca A, Pereira M, et al. Measuring positive mental health in the postpartum period: the bifactor structure of the mental health Continuum-short form in Portuguese women. Assessment 2021;28:1434-44.

38 Reise SP. Invited paper: the rediscovery of Bifactor measurement models. Multivariate Behav Res 2012;47:667-96.

39 Rios J, Wells $\mathrm{C}$. Validity evidence based on internal structure. Psicothema 2014;26:108-16.

40 Reise SP, Scheines R, Widaman KF, et al. Multidimensionality and structural coefficient bias in structural equation modeling. Educ Psychol Meas 2013;73:5-26.

41 BifactorlndicesCalculator. Index of /src/contrib/archive/ bifactorindicescalculator, 2020. Available: https://cran.r-project.org/ src/contrib/Archive/BifactorlndicesCalculator/

42 Chen FF. Sensitivity of goodness of fit indexes to lack of measurement invariance. Structural Equation Modeling: A Multidisciplinary Journal 2007;14:464-504.

43 Santesson AHE, Bäckström M, Holmberg R, et al. Confirmatory factor analysis of the evidence-based practice attitude scale (EBPAS) in a large and representative Swedish sample: is the use of the total scale and subscale scores justified? BMC Med Res Methodol 2020;20:254.

44 Minichilli F, Gorini F, Ascari E, et al. Annoyance judgment and measurements of environmental noise: a focus on Italian secondary schools. Int J Environ Res Public Health 2018;15. doi:10.3390/ijerph15020208. [Epub ahead of print: 26012018 ]. 